\title{
Standard-dose osimertinib for refractory leptomeningeal metastases in T790M- positive EGFR-mutant non-small cell lung
}

\section{cancer}

\author{
Shigeki Nanjo ${ }^{1}$, Akito Hata*,1, Chiyuki Okuda ${ }^{1}$, Reiko Kaji ${ }^{1}$, Hideaki Okada ${ }^{2}$, Daisuke Tamura ${ }^{3}$, Kei Irie ${ }^{4}$, \\ Hiroshi Okada ${ }^{4}$, Shoji Fukushima ${ }^{5}$ and Nobuyuki Katakami ${ }^{1}$ \\ ${ }^{1}$ Division of Integrated Oncology, Institute of Biomedical Research and Innovation Hospital, 2-2, Minatojima-minamimachi, Chuo- \\ ku, Kobe 650-0047, Japan; ${ }^{2}$ Department of Medical Oncology, Kobe Minimally Invasive Cancer Center, Kobe 650-0046, Japan; \\ ${ }^{3}$ Department of Respiratory Medicine, Kobe University Hospital, Kobe 650-0017, Japan; ${ }^{4}$ Department of Pharmacology, Institute of \\ Biomedical Research and Innovation Hospital, Kobe 650-0047, Japan and ${ }^{5}$ Faculty of Pharmaceutical Science, Department of \\ Pharmaceutics, Kobe Gakuin University, Kobe 650-8586, Japan
}

Background: Osimertinib demonstrated promising efficacy for refractory leptomeningeal metastases (LM) in preclinical data and a clinical study at $160 \mathrm{mg}$, but there is limited data for the standard $80 \mathrm{mg}$ dose.

Methods: T790M-positive patients with suspected LM after classical epidermal growth factor receptor-tyrosine kinase inhibitor (EGFR-TKI) failure were enroled.

\begin{abstract}
Results: We investigated 13 patients (5 definitive and 8 possible LM cases). In two of the five definitive cases with T790M in and outside the central nervous system (CNS), osimertinib was effective for both lesions, with cerebrospinal fluid (CSF) clearance of cancer cells and sensitive/T790M mutations. In three definitive cases with extra-CNS T790M without CSF T790M, cancer cells and sensitive mutations in the CSF persisted after osimertinib initiation. The median progression-free survival of all 13 patients was 7.2 months. Osimertinib was generally well-tolerated despite poor performance status, but interstitial lung disease (grade 2) was confirmed in one patient. Based on 25 samples from 13 patients, the osimertinib CSF penetration rate was $2.5 \pm 0.3 \%$.
\end{abstract}

Conclusions: Osimertinib $80 \mathrm{mg}$ is a useful therapeutic option for refractory LM after classical EGFR-TKI failure. It appears more effective in CSF T790M-positive cases.

Epidermal growth factor receptor-tyrosine kinase inhibitors (EGFR-TKIs) show impressive effectiveness for patients with non-small cell lung cancer (NSCLC) harbouring EGFR-sensitive mutations (Lynch et al, 2004; Paez et al, 2004). Despite an initial dramatic response, most cancer cells that respond to these TKIs acquire resistance. Several mechanisms have been identified and the 'gatekeeper' EGFR mutation, a threonine-to-methionine substitution at amino acid position 790 in exon 20 (T790M), is the most common and accounts for more than half of acquired resistance cases ( $\mathrm{Yu}$ et al, 2013). To overcome T790M-mediated resistance, third-generation EGFR-TKIs have been developed. Among them, osimertinib has demonstrated remarkable efficacy for patients with T790M resistant to classical EGFR-TKIs (Jänne et al, 2015; Mok et al, 2017).

*Correspondence: Dr A Hata; E-mail: a-hata@fbri.org

Received 29 April 2017; revised 28 September 2017; accepted 9 October 2017; published online 30 November 2017

(C) 2018 Cancer Research UK. All rights reserved 0007-0920/18 
Central nervous system (CNS) metastases, especially leptomeningeal metastases (LM), are associated with poor prognosis in NSCLC (Hata et al, 2015). Although radiation therapies such as stereotactic radiosurgery or whole-brain radiotherapy (WBRT) are indicated for solitary or multiple brain metastases, they are not associated with prognosis in patients with LM (Morris et al, 2012). Epidermal growth factor receptor-TKIs are initially sensitive to CNS metastases in early clinical course (Park et al, 2012), whereas there are few therapeutic options for refractory LM after failure of classical EGFR-TKIs. High-dose EGFR-TKIs were investigated for such cases, but their effects were only moderate (Hata et al, 2011;

\section{Table 1. Patient characteristics}

\begin{tabular}{|l|c|}
\hline Characteristic & Number (\%) \\
\hline Age & $67(54-79)$ \\
\hline Median (range) & $5(38 \%)$ \\
\hline Sex & $8(62 \%)$ \\
\hline Male & $7(54 \%)$ \\
Female & $6 / 0(46 \%)$ \\
\hline Smoking history & $13(100 \%)$ \\
\hline Never \\
Former/current \\
\hline Histology & $10(77 \%)$ \\
\hline Adenocarcinoma & $3(23 \%)$ \\
\hline Primary EGFR-sensitive mutations \\
\hline Del-19 \\
L858R & $4(3-8)$ \\
\hline Prior regimens & $2(1-3)$ \\
\hline Median (range) & $3(23 \%)$ \\
\hline Number of prior EGFR-TKls & $10(77 \%)$ \\
\hline Median (range) & $7(54 \%)$ \\
\hline Prior whole-brain radiotherapy & $6(46 \%)$ \\
\hline Irradiated & \\
None & \\
\hline Anti-brain edema therapy & \\
\hline Steroids/glycerol & \\
None & \\
\hline Abbreviations: EGFR=epidermal growth factor receptor; TKI=tyrosine kinase inhibitor. \\
\hline
\end{tabular}

Grommes et al, 2011). Notably, osimertinib has promising efficacy for refractory LM in preclinical data and a clinical study at $160 \mathrm{mg}$ (Nanjo et al, 2016 and Yang et al, 2017), but there are limited clinical data at the $80 \mathrm{mg}$ globally approved standard dosage.

\section{MATERIALS AND METHODS}

Patients. This was a prospective pilot study to evaluate the efficacy and safety of clinical standard-dose osimertinib $(80 \mathrm{mg})$ for refractory LM in T790M-positive EGFR-mutant NSCLC patients after failure of standard-dose EGFR-TKIs. The cerebrospinal fluid (CSF) penetration rate of osimertinib and CSF-EGFR mutational status were also investigated. T790M-positive patients with suspected LM after classical EGFR-TKI failure were enroled. We defined: (1) definitive cases as having confirmed cancer cells and EGFR mutations in CSF, and (2) possible cases as continuously unconfirmed cancer cells and mutations in CSF, but suspected LM by radiographical and/or neurological findings. Informed consent was obtained by all enroled patients. The study was approved by the institutional review board and complied with the Declaration of Helsinki.

Evaluation of efficacy and safety. Two to 4 weeks after initiation of $80 \mathrm{mg}$ osimertinib, brain magnetic resonance imaging (MRI) and lumbar puncture were routinely performed, following that, chest/abdominal computed tomography, brain MRI, and lumbar puncture were performed every 1-3 months. The progression-free survival (PFS) of osimertinib therapy was estimated based on a systemic (intra-/extra-CNS disease mixed) evaluation. Radiographical, cytological, neurological, and EGFR mutational findings were regularly evaluated. Neurological changes were evaluated by the following factors: disorientation (date and time, location, and name), headache, diplopia, blindness, paraesthesia, gait disturbance, and grip strength. We also performed the finger-nose test, eye movement test, meningeal sign test, Barre test, and sense of touch test. Extra-CNS response was evaluated according to the Response Evaluation Criteria in Solid Tumours (RECIST) version 1.1. As CNS radiographic changes are difficult to assess by the RECIST, they were evaluated as improved, stable, and progressed based on findings of dura mater thickening, exuding contrast agent, ventricular distention, and/or, concomitant substantial brain metastases with confirmation by at least two doctors. Adverse

Table 2. CSF mutational and therapeutic results

\begin{tabular}{|c|c|c|c|c|c|c|c|}
\hline Patient & $\begin{array}{l}\text { CSF-sensitive } \\
\text { mutation }\end{array}$ & $\begin{array}{c}\text { CSF } \\
\text { T790M }\end{array}$ & PS & $\begin{array}{c}\text { Neurological } \\
\text { Findings } \rightarrow \text { Change }\end{array}$ & $\begin{array}{c}\text { CNS radiographic } \\
\text { change }\end{array}$ & $\begin{array}{c}\text { Extra-CNS rebiopsy site/ } \\
\text { T790M status }\end{array}$ & $\begin{array}{c}\text { Extra-CNS } \\
\text { response }\end{array}$ \\
\hline 1 & L858R & + & $3 \rightarrow 1$ & Abnormal $\rightarrow$ Improved & Improved & Bone/+ & PR \\
\hline 2 & Del-19 & + & $3 \rightarrow 1$ & Abnormal $\rightarrow$ Improved & Improved & Lung/ + & NE \\
\hline 3 & Del-19 & - & $3 \rightarrow 3$ & Abnormal $\rightarrow$ Stable & Stable & Liver/ + & PR \\
\hline 4 & Del-19 & - & $2 \rightarrow 2$ & Abnormal $\rightarrow$ Stable & Stable & Lymph node/ + & NE \\
\hline 5 & L858R & - & $3 \rightarrow 4$ & Abnormal $\rightarrow$ Worsened & Progressed & Lung/ + & SD \\
\hline 6 & - & - & $1 \rightarrow 1$ & Normal $\rightarrow$ Stable & Improved & Pleura/ + & PR \\
\hline 7 & - & - & $2 \rightarrow 1$ & Normal $\rightarrow$ Stable & Stable & Pleura/ + & SD \\
\hline 8 & - & - & $1 \rightarrow 1$ & Normal $\rightarrow$ Stable & Improved & Lung/ + & PR \\
\hline 9 & - & - & $1 \rightarrow 1$ & Normal $\rightarrow$ Stable & Improved & Lung/ + & PR \\
\hline 10 & - & - & $1 \rightarrow 1$ & Abnormal $\rightarrow$ Improved & Improved & Lung/ + & PR \\
\hline 11 & - & - & $3 \rightarrow 1$ & Abnormal $\rightarrow$ Improved & Improved & Liver/ + & SD \\
\hline 12 & - & - & $1 \rightarrow 1$ & Normal $\rightarrow$ Stable & Improved & Pleura/ + & PR \\
\hline 13 & - & - & $1 \rightarrow 1$ & Normal $\rightarrow$ Stable & NE & Lung/+ & PR \\
\hline
\end{tabular}


A

Progression-free survival (months)

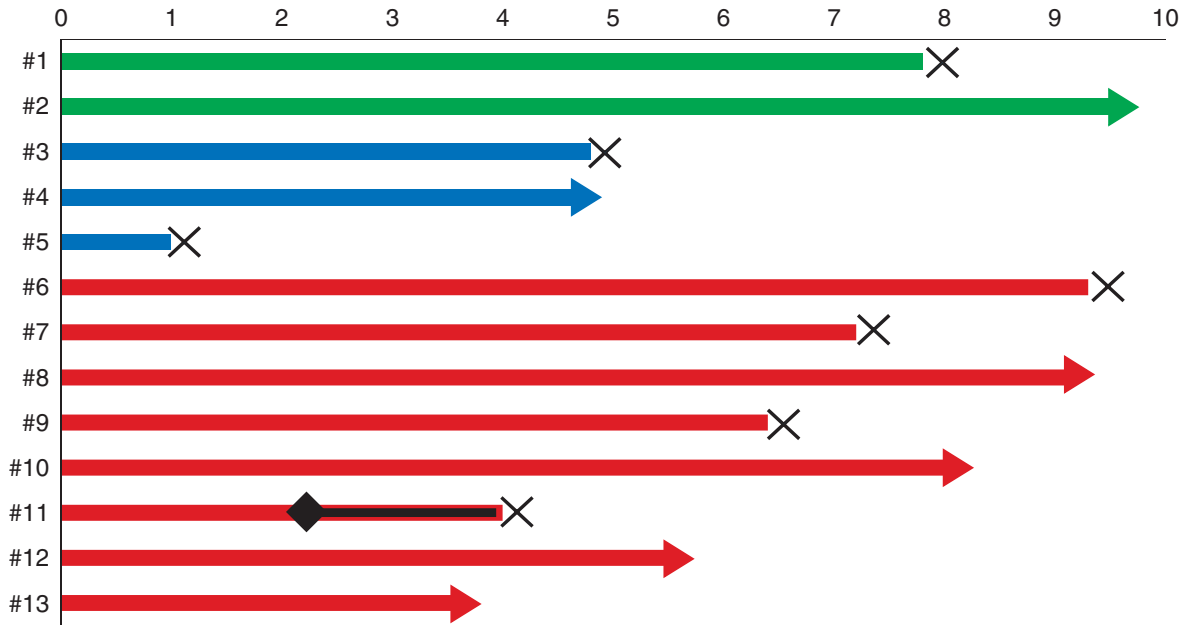

CSF-T790M (+) definitive cases $\quad$ X Progressive disease $\quad$ CSF, cerebrospinal fluid
CSF-T790M (-) definitive cases

CSF-T790M (-) definitive cases

Possible cases

Interstitial lung disease

B

Lumbar puncture before osimertinib

Progression-free survival (months)

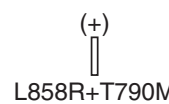

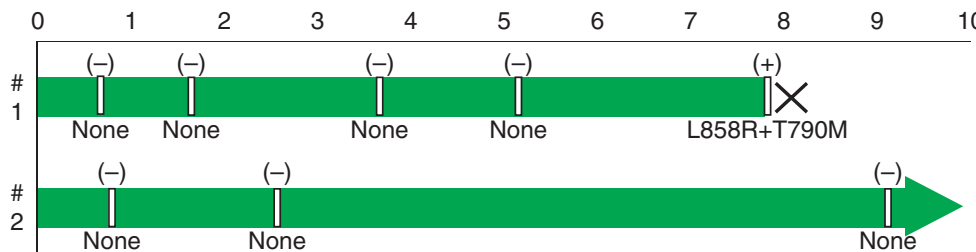

Del-19+T790M

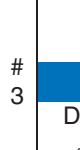

(+) (+)

$\prod_{\text {Del-19 }}^{(+)}$

$\prod_{\text {Del-19 }}^{(+)}$

L858R

Del-19 Del-19
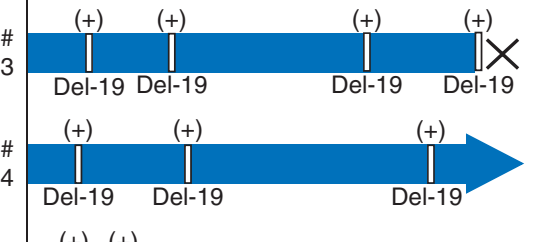

\#

CSF-T790M (+) cases

CSF-T790M (-) cases

Х Progressive disease

I Lumbar puncture

(+) CSF cytology positive

(-) CSF cytology negative
CSF, cerebrospinal fluid; LM,

leptomeningeal metastases

Figure 1. Treatment and CSF results. (A) Treatment timelines. (B) Cerebrospinal fluid (CSF) cytology/mutation status in definitive LM cases.

events (AEs) were evaluated based on the National Cancer Institute-Common Toxicity Criteria, version 4.0.

Epidermal growth factor receptor mutational analyses and quantitative analyses of osimertinib. We isolated DNA from each tumour specimen by sampling extra-CNS lesions and CSF. EGFR-sensitive and T790M mutations were analysed using highly sensitive assays: the peptide nucleic acid-locked nucleic acid PCR clamp method or the mutation-biased PCR quenching probe method (Nagai et al, 2005; Nakamura et al, 2011).

All CSF samples were collected after $6 \pm 2 \mathrm{~h}$ from osimertinib administration and plasma samples were simultaneously collected. Cerebrospinal fluid and plasma concentrations of osimertinib were measured using liquid chromatography-tandem mass spectrometry (LC-MS/MS). The CSF penetration rate of osimertinib was estimated based on CSF/plasma concentrations.

\section{RESULTS}

Patients. We enroled a total of 13 patients (5 definitive and 8 possible cases) following osimertinib approval in April 2016. Patient characteristics are shown in Table 1 . The median age was 67 (range 54-79). All tumour histology was adenocarcinoma. The types of EGFR-sensitive mutation were as follows: 10 (77\%) deletional mutations in exon 19 (Del-19) and 3 (23\%) L858R point mutations in exon 21. The median numbers of prior regimens and EGFR-TKIs (gefitinib, erlotinib, and/or afatinib) were four (range 3-8) and two (range 1-3), respectively. Prior WBRT was performed in three $(23 \%)$ patients. Seven $(54 \%)$ patients underwent anti-brain edema therapy (steroids/glycerol). No patients received intrathecal chemotherapy, highdose EGFR-TKIs, or third-generation EGFR-TKIs before enrolment. 


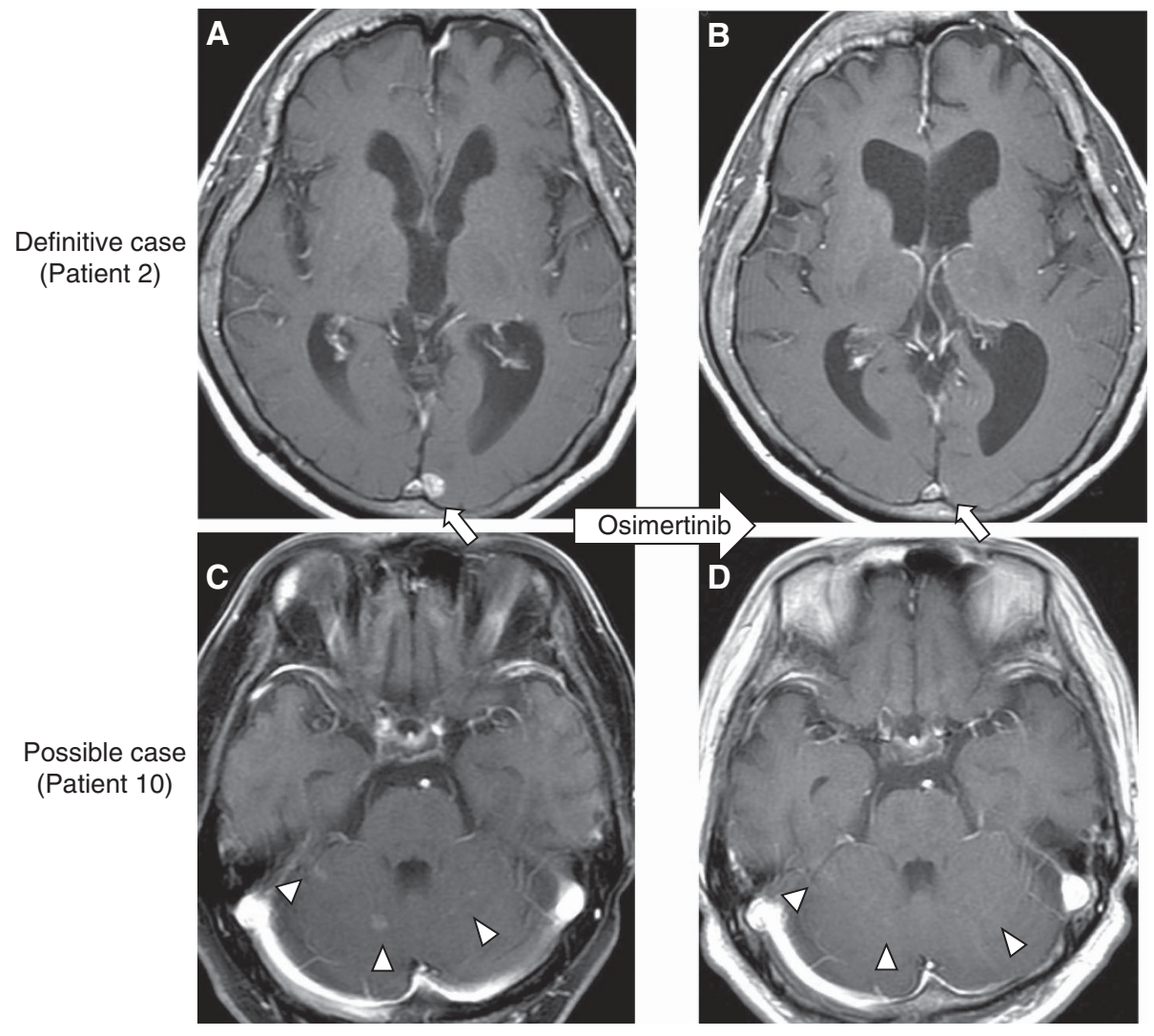

Figure 2. Representative magnetic resonance images before and after osimertinib (patients 2 and 10) Patient 2 (definitive case): (A) before osimertinib and (B) 2 months after osimertinib initiation. Patient 10 (possible case): (C) before osimertinib and (D) 2 months after osimertinib initiation.

Mutation and therapeutic results. Table 2 shows the mutation and therapeutic results. T790M was confirmed by extra-CNS rebiopsy in all 13 cases. In all five definitive cases, malignant cells and sensitive EGFR mutations were detected in CSF, and T790M was confirmed in two of these five patients. Osimertinib was markedly effective in both confirmed T790M CNS and extra-CNS cases (patients 1 and 2). Radiographical improvement, improved performance status (PS), and better neurological findings were also observed. In three definitive cases with T790M outside the CNS but not the CSF (patients 3-5), disease control of both areas were achieved in two cases (patients 3 and 4), whereas osimertinib was ineffective in CNS, despite being effective for lung lesions in another case (patient 5). In all eight possible cases (patient 6-13), disease control was achieved both in and outside the CNS. Central nervous system and extra-CNS improvements were observed in six (75\%) and five (63\%) patients, respectively. Abnormal neurological findings improved in two of two patients (10 and 11). Performance status ameliorated in the two of two patients with poor PS (7 and 11).

Figure 1A depicts treatment times. The median PFS of all 13 patients was 7.2 (95\% confidence interval: 4.0 , undeterminable) months. Progression was confirmed in seven cases and six patients are currently being treated. The PFS was longer in CSF-T790M $(+)$ cases $(9.6+$ and 7.8 months) than in CSF-T790M $(-)$ cases (4.8, 4.7+, and 1.0 months). Median overall survival was not reached. Regarding post-osimertinib therapy, best supportive care only was chosen in $1(8 \%)$ case. Osimertinib was continued beyond progression in four (31\%) cases. Cytotoxic chemotherapy was administered in two (15\%) cases. Whole-brain radiotherapy was performed in three $(23 \%)$ cases where CNS lesions progressed.

Figure 1B shows CSF cytology/mutation status in definitive cases. Cerebrospinal fluid clearance of cancer cells and sensitive/ T790M mutations were confirmed after osimertinib initiation in CSF-T790M $(+)$ cases. In patient 1 (PFS: 7.8 months), cancer cells and L858R + T790M were detected again by lumbar puncture at the time of progression. In patient 2 (PFS: $9.6+$ months), cancer cells and Del-19+ T790M were not detected by lumbar puncture 9 months after osimertinib initiation. In CSF-T790M $(-)$ cases, cancer cells and sensitive mutations in CSF remained positive continuously after osimertinib therapy.

Figure 2 shows representative MRIs before and after osimertinib (patients 2 and 10).

Safety. Ten $(77 \%)$ patients had rash $\leqslant$ grade 2 . Paronychia $\leqslant$ grade 2 was observed in six $(46 \%)$ patients. No $\geqslant$ grade 3 AEs, diarrhoea, or liver dysfunction were observed. Interstitial lung disease (ILD, grade 2) was confirmed in one patient and occurred 2 months after osimertinib initiation. The patient initially complained of dyspnea on effort and productive cough. Clinical and radiographic findings were improved following corticosteroid therapy. There were no dose reductions and o1 dose interruptions due to ILD.

Cerebrospinal fluid penetration rate. Based on 25 samples from 13 patients, CSF and plasma concentrations of osimertinib (mean \pm s.d.) were $14.4 \pm 2.8 \mathrm{~nm}$ and $555.3 \pm 51.5 \mathrm{nM}$, respectively. The corresponding medians were 8.1 (range, 1.6-56.6) $\mathrm{nm}$ and 483.3 (95.4-1267.0) nM. The CSF penetration rate of osimertinib was estimated at $2.5 \pm 0.3 \%$ (mean \pm s.d.) and the median was 2.0 (range 0.5-6.9).

\section{DISCUSSION}

Our study suggested the efficacy and safety of standard-dose osimertinib $(80 \mathrm{mg})$ for refractory LM in T790M-positive EGFRmutant NSCLC patients. It was especially effective in two CSF T790M-positive definitive cases, and CSF clearance of cancer cells and sensitive/T790M mutations was confirmed during response 
continuation. Conversely, CSF cancer cells and sensitive mutations remained continuously positive after osimertinib initiation in three CSF T790M-negative definitive cases. Yang et al (2017) showed the efficacy of high-dose $(160 \mathrm{mg})$ osimertinib for refractory LM in CSF T790M-positive patients (). Notably, CSF clearance was only confirmed in two CSF T790M-positive patients of their study. These results suggest superior efficacy of osimertinib in CSF T790M-positive patients.

One ILD (grade 2) was observed, but osimertinib was generally safe despite poor PS populations with LM. Although afatinib and high-dose erlotinib are potentially effective for refractory LM, their toxicities might be unsuitable for poor PS with LM (Hata et al, 2011; Grommes et al, 2011; Hoffknecht et al, 2015). In two of three CSF T790M-negative definitive cases, osimertinib could control disease for $\sim 5$ months and was well-tolerated without CSF clearance. Thus, osimertinib may be a suitable option for poor PS patients with LM.

Our study results estimated an osimertinib CSF penetration rate of $2.5 \pm 0.3 \%$. Poor EGFR-TKI penetration is a main cause of 'pharmacokinetic failure' in the CNS. Cerebrospinal fluid penetration rates of classical EGFR-TKIs were reported at $0.7-2.8 \%$ (Pareek et al, 2016). Among them, erlotinib is effective for refractory LM (Grommes et al, 2011; Hata et al, 2011). The CSF penetration rate of osimertinib in our study was comparable to that of erlotinib. From a pharmacokinetic perspective, osimertinib may be a reasonable option for LM.

Our study has several limitations. First, it was small sample size. However, it is extremely difficult to conduct a large study of refractory LM patients. In fact, only a few case reports have been published (Takeda et al, 2017). Second, we cannot directly compare $160 \mathrm{mg}$ clinical trial data with our $80 \mathrm{mg}$ results. A previous osimertinib $160 \mathrm{mg}$ trial estimated a higher CSF penetration rate (16\%) (Yang et al, 2017) than our results, and it may be better to deliver the higher dosage to the CNS. However, osimertinib $160 \mathrm{mg}$ is not available in clinical practice, and their penetration rate was based on a different definition. Although previous studies used the same method (LC-MS/MS) and showed similar CSF and plasma concentrations of osimertinib (Planchard et al, 2016; Yang et al, 2017), their study assumed $5.3 \%$ as the ratio of plasma free osimertinib, and adopted plasma free osimertinib as the denominator. We decided to include all plasma osimertinib since no accurate measurement of plasma free osimertinib was available. This dose was effective and safe; 10 of 23 patients showed radiological improvement and all AEs were grade $1 / 2$, except one case of grade 3 diarrhoea and nausea in their study, which is comparable to our findings.

In conclusion, osimertinib at $80 \mathrm{mg}$ has a similar or higher CSF penetration rate compared with classical EGFR-TKIs and is a notable therapeutic option for refractory LM after classical EGFRTKI failure in T790M-positive EGFR-mutant NSCLC patients. It appears more effective in CSF T790M-positive cases. Further studies are warranted to evaluate clinical efficacy of standard-dose osimertinib for refractory LM.

\section{ACKNOWLEDGEMENTS}

We thank Mr David Martin for writing support. The study was partially supported by research assistance funds from the Foundation for Biomedical Research and Innovation.

\section{CONFLICT OF INTEREST}

$\mathrm{AH}$ received lecture fees from Chugai, Astra Zeneca, Boeringer Ingelheim, and Eli Lilly. NK received grants from Astra Zeneca,
Eisai, Ono, Kyowa Kirin, Shionogi, Daiichi-Sankyo, Taiho, Chugai, Eli Lilly, Boeringer Ingelheim, and Merck Serono, and payment for lectures from Dainippon Sumitomo, Chugai, Boeringer Ingelheim, Astra Zeneca, Eli Lilly, Taiho, Janssen, Novartis, Pfizer, Ono, and Daiichi-Sankyo. All other authors declare no conflict of interest.

\section{REFERENCES}

Grommes C, Oxnard GR, Kris MG, Miller VA, Pao W, Holodny AI, Clarke JL, Lassman AB (2011) 'Pulsatile' high-dose weekly erlotinib for CNS metastases from EGFR mutant non-small cell lung cancer. Neuro Oncol 13: $1364-1369$.

Hata A, Kaji R, Fujita S, Katakami N (2011) High-dose erlotinib for refractory brain metastases in a patient with relapsed non-small cell lung cancer. J Thorac Oncol 6: 653-654.

Hata A, Katakami N, Yoshioka H, Takeshita J, Tanaka K, Masago K, Fujita S, Kaji R, Imai Y, Monden K, Matsumoto T, Nagata K, Otsuka K, Tachikawa R, Tomii K, Kunimasa K, Iwasaku M, Nishiyama A, Ishida T, Nishimura Y (2015) Prognostic impact of central nervous system metastases after acquired resistance to EGFR-TKI: poorer prognosis associated with T790M-negative status and leptomeningeal metastases. Anticancer Res 35: 1025-1031.

Hoffknecht P, Tufman A, Wehler T, Pelzer T, Wiewrodt R, Schütz M, Serke M, Stöhlmacher-Williams J, Märten A, Maria Huber R, Dickgreber NJ (2015) Efficacy of the irreversible ErbB family blocker afatinib in epidermal growth factor receptor (EGFR) tyrosine kinase inhibitor (TKI)-pretreated non-small-cell lung cancer patients with brain metastases or leptomeningeal disease. J Thorac Oncol 10: 156-163.

Jänne PA, Yang JC, Kim DW, Planchard D, Ohe Y, Ramalingam SS, Ahn MJ, Kim SW, Su WC, Horn L, Haggstrom D, Felip E, Kim JH, Frewer P, Cantarini M, Brown KH, Dickinson PA, Ghiorghiu S, Ranson M (2015) AZD9291 in EGFR inhibitor-resistant non-small-cell lung cancer. $N$ Engl J Med 372: 1689-1699.

Lynch TJ, Bell DW, Sordella R, Gurubhagavatula S, Okimoto RA, Brannigan BW, Harris PL, Haserlat SM, Supko JG, Haluska FG, Louis DN, Christiani DC, Settleman J, Haber DA (2004) Activating mutations in the epidermal growth factor receptor underlying responsiveness of non-smallcell lung cancer to gefitinib. N Engl J Med 350: 2129-2139.

Mok TS, Wu Y-L, Ahn M-J, Garassino MC, Kim HR, Ramalingam SS, Shepherd FA, He Y, Akamatsu H, Theelen WS, Lee CK, Sebastian M, Templeton A, Mann H, Marotti M, Ghiorghiu S, Papadimitrakopoulou VA, AURA3 Investigators (2017) Osimertinib or platinum-pemetrexed in EGFR T790M-positive lung cancer. N Engl J Med 376: 629-640.

Morris PG, Reiner AS, Szenberg OR, Clarke JL, Panageas KS, Perez HR, Kris MG, Chan TA, DeAngelis LM, Omuro AM (2012) Leptomeningeal metastasis from non-small cell lung cancer: survival and the impact of whole brain radiotherapy. J Thorac Oncol 7: 382-385.

Nagai Y, Miyazawa H, Huqun, Tanaka T, Udagawa K, Kato M, Fukuyama S, Yokote A, Kobayashi K, Kanazawa M, Hagiwara K (2005) Genetic heterogeneity of the epidermal growth factor receptor in non-small cell lung cancer cell lines revealed by a rapid and sensitive detection system, the peptide nucleic acid-locked nucleic acid PCR clamp. Cancer Res 65, 7276-728205-0331.

Nakamura T, Sueoka-Aragane N, Iwanaga K, Sato A, Komiya K, Abe T, Ureshino N, Hayashi S, Hosomi T, Hirai M, Sueoka E, Kimura S (2011) A noninvasive system for movnitoring resistance to epidermal growth factor receptor tyrosine kinase inhibitors with plasma DNA. J Thorac Oncol 6: 1639-1648.

Nanjo S, Ebi H, Arai S, Takeuchi S, Yamada T, Mochizuki S, Okada Y, Nakada M, Murakami T, Yano S (2016) High efficacy of third generation EGFR inhibitor AZD9291 in a leptomeningeal carcinomatosis model with EGFR-mutant lung cancer cells. Oncotarget 7: 3847-3856.

Paez JG, Jänne PA, Lee JC, Tracy S, Greulich H, Gabriel S, Herman P, Kaye FJ, Lindeman N, Boggon TJ, Naoki K, Sasaki H, Fujii Y, Eck MJ, Sellers WR, Johnson BE, Meyerson M. (2004) EGFR mutations in lung cancer: correlation with clinical response to gefitinib therapy. Science 304: 1497-1500.

Pareek V, Welch M, Ravera E, Zampolin RL, Sequist LV, Halmos B (2016) Marked Differences in CNS Activity among EGFR Inhibitors: Case Report and Mini-Review. J Thorac Oncol 11: e135-e139.

Park SJ, Kim HT, Lee DH, Kim KP, Kim SW, Suh C, Lee JS (2012) Efficacy of epidermal growth factor receptor tyrosine kinase inhibitors for brain 
metastasis in non-small cell lung cancer patients harboring either exon 19 or 21 mutation. Lung Cancer 77: 556-560.

Planchard D, Brown KH, Kim DW, Kim SW, Ohe Y, Felip E, Leese P, Cantarini M, Vishwanathan K, Jänne PA, Ranson M, Dickinson PA (2016) Osimertinib Western and Asian clinical pharmacokinetics in patients and healthy volunteers: implications for formulation, dose, and dosing frequency in pivotalclinical studies. Cancer Chemother Pharmacol 77: $767-776$.

Takeda T, Itano H, Takeuchi M, Nishimi Y, Saitoh M, Takeda S (2017) Osimertinib administration via nasogastric tube in an EGFR-T790Mpositive patient with leptomeningeal metastases. Respirol Case Rep 5: e00241.

Yang JC, Cho B, Kim DW, Kim SW, Lee JS, Su WC, John T, Kao CH, Natale R, Goldman JW, Overend P, Vishwanathan K, Ye X, Yang Z, Ahn MJ (2017) Osimertinib for patients (pts) with leptomeningeal metastases (LM) from EGFR-mutant non-small cell lung cancer (NSCLC): Updated results from BLOOM study. J Clin Oncol 35(suppl): abstr 2020.

Yu HA, Arcila ME, Rekhtman N, Sima CS, Zakowski MF, Pao W, Kris MG, Miller VA, Ladanyi M, Riely GJ (2013) Analysis of tumor specimens at the time of acquired resistance to EGFR-TKI therapy in 155 patients with EGFR-mutant lung cancers. Clin Cancer Res 19: $2240-2247$.

This work is published under the standard license to publish agreement. After 12 months the work will become freely available and the license terms will switch to a Creative Commons AttributionNonCommercial-Share Alike 4.0 Unported License. 